\title{
Identification of Potential Prognostic Long Non-Coding RNA Biomarkers for Predicting Recurrence in Patients with Cervical Cancer
}

This article was published in the following Dove Press journal: Cancer Management and Research

\begin{abstract}
Yan Zhang,' Xing Zhang,' Haixia Zhu, ${ }^{2}$ Yang Liu, ${ }^{3}$ Jian Cao, ${ }^{3}$ Dake Li, ${ }^{3}$ Bo Ding, ${ }^{4}$ Wenjing Yan, ' Hua Jin, 2,* Shizhi Wang (D) ${ }^{1, *}$

'Key Laboratory of Environmental Medicine Engineering, Ministry of Education, School of Public Health, Southeast University, Nanjing, People's Republic of China; ${ }^{2}$ Clinical Laboratory, Affiliated Tumor Hospital of Nantong University (Nantong Tumor Hospital), Nantong, People's Republic of China; ${ }^{3}$ Department of Gynecology, Women's Hospital of Nanjing Medical University, Nanjing Maternity and Child Health Care Hospital, Nanjing, People's Republic of China; ${ }^{4}$ Department of Gynecology and Obstetrics, Zhongda Hospital, School of Medicine, Southeast University, Nanjing, People's Republic of China
\end{abstract}

*These authors contributed equally to this work

Correspondence: Shizhi Wang

Key Laboratory of Environmental

Medicine Engineering, Ministry of

Education, School of Public Health,

Southeast University, 87 Dingjiaqiao,

Gulou District, Nanjing 210009, People's

Republic of China

Tel +862583272566

Fax +86 2583324322

Email shizhiwang2009@seu.edu.cn

Hua Jin

Clinical Laboratory, Affiliated Tumor Hospital of Nantong University (Nantong Tumor Hospital), No. 30, North Tongyang

Road, Tongzhou District, Nantong

22636I, People's Republic of China

Tel +8651389002658

Fax +8651385169100

Email ntmgjh@I63.com
Background: Cervical cancer (CC) is one of the most common malignant tumors in women, and its treatment is often accompanied by high recurrence. We aimed to identify the long non-coding RNAs (lncRNAs) associated with CC recurrence.

Methods: We downloaded lncRNAs expression data of $\mathrm{CC}$ patients from The Cancer Genome Atlas (TCGA) dataset and used Cox regression models to analyze the lncRNAs relationship with $\mathrm{CC}$ recurrence. The significantly associated lncRNAs were utilized to construct a recurrence risk score (RRS) model. Bioinformatics analyses were used to assess the potential role of the critical lncRNAs in CC recurrence. The effect of critical lncRNAs on $\mathrm{CC}$ phenotype was determined by in vitro experiments.

Results: Using Cox regression analysis, four lncRNAs, ie, HCG11, CASC15, LINC00189, and LINC00905, were markedly associated with worse recurrence-free survival (RFS) of CC, whereas three lncRNAs, including HULC, LINC00173, and MIR22HG, were the opposite. After constructing the RRS model, Kaplan-Meier analysis revealed that patients with high RRS had significantly increased risk of recurrence. Among the 20 types of tumors in the TCGA database which all had adjacent normal tissues, MIR22HG and HCG11were significantly downregulated in 18 and 10 types of tumors including CC, respectively. Increased MIR22HG was significantly relevant to decreased risks of recurrence among the subgroups of age at diagnosis $<45($ Hazard Ratio $(H R)=0.26)$, stage I/II $(H R=0.33)$, T stage I/II $(H R=0.30)$, chemotherapy $(\mathrm{HR}=0.18)$, and molecular therapy $(\mathrm{HR}=0.16)$. Functionally, elevated MIR22HG expression could suppress CC cell proliferation, migration and invasion.

Conclusion: MIR22HG has a fundamental role in $\mathrm{CC}$ recurrence and could be served as a potential prognostic biomarker.

Keywords: TCGA, IncRNAs, cervical cancer, recurrence, biomarker

\section{Introduction}

Cervical cancer (CC) is one of the most common malignant tumors in women. The global cancer statistics demonstrate that $\mathrm{CC}$ is the fourth most common cancer in females, with an annual incidence of about 530,000 new cases and a death toll of $270,000{ }^{1}$ With the development of the prophylactic HPV vaccination and CC screening, the 5-year overall survival (OS) rate of $\mathrm{CC}$ has been prolonged. ${ }^{2}$ However, in many developing countries, the 3- to 5-year OS rate of CC is less than 50\%; especially in patients with advanced disease, the recurrence rate of CC is as high as $70 \% .^{3,4}$ The cure rate for recurrent $\mathrm{CC}$ is very low, and the 5 -year survival rate for relapsed patients is less than $5 \%$. According to the International 
Federation of Obstetrics and Gynecology (FIGO), the recurrence rate of $\mathrm{CC}$ is $11 \%$ to $22 \%$ and $28 \%$ to $64 \%$ for the FIGO stage IB-IIA and IIB-IVA, respectively. ${ }^{5}$ The prognosis of recurrent $\mathrm{CC}$ is poor, the treatment time is limited and the quality of life deteriorates rapidly. The treatment of recurrent $\mathrm{CC}$ remains challenging. ${ }^{6}$ Therefore, it is necessary to find new biomarkers related to $\mathrm{CC}$ recurrence and provide evidence for prevention and personalized treatment of $\mathrm{CC}$ recurrence.

Long non-coding RNAs (lncRNAs) are a kind of long RNA transcripts, which are longer than 200 nucleotides and unable to encode proteins. ${ }^{7}$ With the development of bioinformatics and the application of a series of new experimental methods such as high throughput sequencing, gene chip, and genome enrichment analysis, more and more lncRNAs have been found. ${ }^{8}$ Some of the dysregulated IncRNAs, involving the regulation of multiple cellular pathways, have revealed oncogenic and tumor-suppressive roles in cancer development, progression, and metastasis. ${ }^{9}$ Accumulating evidence has demonstrated that some specific lncRNAs are abnormally expressed in CC cells and participate in gene regulation, such as reducing or enhancing the expression of target genes in the process of carcinogenesis at the transcriptional level, and blocking or promoting the development of cancer. ${ }^{10}$ Much work so far had reported that lncRNAs can be used as biomarkers for $\mathrm{CC}$ invasion, metastasis and prognosis. ${ }^{11}$ Some lncRNAs were significantly associated with $\mathrm{CC}$ recurrence, such as HOTAIR, snaR and MEG3. ${ }^{12-14}$ LncRNAs provide new insights into the molecular mechanisms and treatments of cancer, and thus lay the ground work for the clinic.

In this study, we used The Cancer Genome Atlas (TCGA) data to identify lncRNAs associated with CC recurrence by a variety of data mining and bioinformatics methods. The identified lncRNAs have the potential to be biomarkers for the prediction of $\mathrm{CC}$ recurrence.

\section{Materials and Methods}

\section{TCGA Database and Patient Information}

The expression and clinical data of $310 \mathrm{CC}$ patients were downloaded from TCGA database (up to December 18, 2018). The inclusion criteria were as follows: 1) patients with pathological diagnosis of primary $\mathrm{CC} ; 2$ ) patients with gene expression and clinical features; 3 ) patients with information of recurrence outcomes. Overall, a total of $211 \mathrm{CC}$ patients with corresponding clinical features such as diagnosing age, metastasis, lymph node status, stage, $\mathrm{T}$ stage, and histological type were enrolled in this study. Data processing procedures also complied with TCGA data and human subject protection policies (www.cancer.gov/about-nci/organization/ ccg/research/structural-genomics/tcga).

\section{Differential Analysis of Expressed IncRNAs}

To investigate the role of IncRNAs in CC recurrence, we searched the annotated IncRNAs on the website of HUGO gene nomenclature committee (HGNC), and a total of 4072 lncRNAs were obtained from the HGNC database (http:// www.genenames.org/cgi-bin/statistics, up to February 19, 2019). The $4072 \operatorname{lncRNAs}$ were searched for expression data in cervical squamous cell carcinoma (CSCC) (TCGA provisional) using the geno/transcriptomic analyses tool of the online bioinformatics website the cBioPortal for Cancer Genomics (hereinafter referred to as cBioPortal; http://www. cbioportal.org/); ${ }^{15}$ finally, there were 471 known lncRNAs with copy number variations in more than $1 \%$ of queried patients. Of the $471 \mathrm{lncRNAs}, 248$ lncRNAs met the criterion of Z-score with a threshold of \pm 2 and expression alteration in more than $1 \%$ CC patients. Genome-wide lncRNAs expression profiles were obtained from the RNA sequencing (RNAseq) dataset of TCGA. The differentially expressed IncRNAs (DELs) were analyzed by R program (https://www.r-project. org/) using the limma package (http://www.bioconductor.org/ packages/release/bioc/html/limma.html. org/packages/release/ bioc/html/limma.html website). The fold change (FC) in the expression of individual IncRNAs was calculated and DELs with $\log 2|\mathrm{FC}|>1.0$ and $P<0.05$ were selected.

\section{Identifying IncRNAs Predictive of Recurrence-Free Survival (RFS)}

To identify lncRNAs predictive of RFS, univariate and multivariate Cox regression analyses were performed to assess the relationship between expression of IncRNAs level and RFS. Then, we constructed a prognostic risk score, ie, recurrence risk score (RRS), for predicting RFS by using the regression coefficient $(\beta)$ from the multivariate Cox regression model as following:

$$
\mathrm{RRS}=\sum_{i=1}^{n}(\operatorname{EXP}(\mathrm{i}) * \beta(\mathrm{i}))
$$

where $\mathrm{n}$ is the number of selected lncRNAs and EXP is the expression level of individual lncRNAs. ${ }^{16}$

According to the above formula, RRS of each patient was calculated, and patients were divided into high- and low-risk groups based on the median of RRS. KaplanMeier (K-M) analysis was applied to evaluate the different 
Table I Demographic and Clinical Characteristics of CC Patients and Their Relationship with RFS of CC

\begin{tabular}{|c|c|c|c|}
\hline \multirow[t]{2}{*}{ Variables } & \multirow[t]{2}{*}{ Case (n\%) } & \multicolumn{2}{|c|}{ Relapse Free Survival } \\
\hline & & HR $(95 \% \mathrm{Cl})$ & $P$ value \\
\hline \multicolumn{4}{|l|}{ Age at diagnosis } \\
\hline$<45$ & $105(49.8 \%)$ & Reference & \\
\hline$\geq 45$ & $106(50.2 \%)$ & $1.70(0.73-3.94)$ & 0.216 \\
\hline \multicolumn{4}{|l|}{ Number of pregnancies } \\
\hline$\leq 3$ & $109(51.7 \%)$ & Reference & \\
\hline$>3$ & 81 (38.4\%) & $0.87(0.38-2.02)$ & 0.752 \\
\hline NA & 21 (9.9\%) & & \\
\hline \multicolumn{4}{|l|}{ Smoking status } \\
\hline No & $104(49.3 \%)$ & Reference & \\
\hline Yes & 91 (43.1\%) & $0.73(0.31-1.70)$ & 0.461 \\
\hline NA & $16(7.6 \%)$ & & \\
\hline \multicolumn{4}{|l|}{ Histological type } \\
\hline Cervical Squamous Cell & 177 (83.9\%) & Reference & \\
\hline \multicolumn{4}{|l|}{ Carcinoma } \\
\hline Others & $34(16.1 \%)$ & $1.10(0.38-3.22)$ & 0.858 \\
\hline \multicolumn{4}{|l|}{ Neoplasm histologic grade } \\
\hline $\mathrm{Gl} / \mathrm{G} 2$ & III (52.6\%) & Reference & \\
\hline G3/G4 & 81 (38.4\%) & $1.47(0.67-3.22)$ & 0.340 \\
\hline GX & $17(8.1 \%)$ & & \\
\hline NA & $2(0.9 \%)$ & & \\
\hline \multicolumn{4}{|l|}{ Stage } \\
\hline 1/II & $167(79.1 \%)$ & Reference & \\
\hline III/IV & $39(18.5 \%)$ & $0.62(0.19-2.0709)$ & 0.436 \\
\hline NA & $5(2.4 \%)$ & & \\
\hline \multicolumn{4}{|l|}{ T stage } \\
\hline $\mathrm{TI} / \mathrm{T} 2$ & 149 (70.6\%) & Reference & \\
\hline $\mathrm{T} 3 / \mathrm{T} 4$ & $12(5.7 \%)$ & $1.28(0.30-5.54)$ & 0.739 \\
\hline $\mathrm{TX}$ & $16(7.6 \%)$ & & \\
\hline NA & $34(16.1 \%)$ & & \\
\hline \multicolumn{4}{|l|}{ Lymph node metastasis } \\
\hline No & 99 (46.9\%) & Reference & \\
\hline NI & $4 \mathrm{I}(19.4 \%)$ & $3.47(1.32-9.13)$ & 0.012 \\
\hline $\mathrm{NX}$ & 37 (17.5\%) & & \\
\hline NA & $34(16.1 \%)$ & & \\
\hline \multicolumn{4}{|l|}{ Metastasis } \\
\hline Mo & $87(41.2 \%)$ & Reference & \\
\hline MI & $9(4.3 \%)$ & $0.05(0.00-3429.88)$ & 0.589 \\
\hline$M X$ & $80(37.9 \%)$ & & \\
\hline NA & $35(16.6 \%)$ & & \\
\hline \multicolumn{4}{|l|}{ Chemotherapy } \\
\hline NO & $110(52.1 \%)$ & Reference & \\
\hline YES & $101(47.9 \%)$ & $1.01(0.46-2.23)$ & 0.968 \\
\hline \multicolumn{4}{|l|}{ Radiation therapy } \\
\hline NO & 61 (28.9\%) & Reference & \\
\hline YES & $136(64.5 \%)$ & $1.00(0.42-2.39)$ & 0.997 \\
\hline NA & $14(6.6 \%)$ & & \\
\hline
\end{tabular}

(Continued)
Table I (Continued).

\begin{tabular}{|l|l|l|l|}
\hline Variables & Case (n\%) & \multicolumn{2}{|l|}{ Relapse Free Survival } \\
\cline { 3 - 4 } & & HR (95\% Cl) & P value \\
\hline Molecular_therapy & & & \\
NO & $56(26.5 \%)$ & Reference & \\
YES & $101(47.9 \%)$ & $0.72(0.31-1.67)$ & 0.439 \\
NA & $54(25.6 \%)$ & & \\
\hline
\end{tabular}

Notes: Bold numbers indicate statistically significant differences between groups, with a $p$ value $<0.05$.

Abbreviations: $\mathrm{CC}$, cervical cancer; RFS, relapse-free survival; NA, non-available.

survival rates between patients with low- and high-RRSs, and Log rank test was used to evaluate the differences between them. The sensitivity and specificity of the RRS were estimated by time-dependent receiver operating characteristic (ROC) curves. To further analyze the association between RFS and clinical features, we carried out subgroup survival analyses to estimate the influence of clinical parameters on the RFS of CC patients.

\section{Functional Enrichment Analysis}

To investigate the biological roles of the candidate lncRNAs signature in $\mathrm{CC}$, we obtained potential co-expressed genes of selected lncRNAs using circlncRNAnet (http://app.cgu. edu.tw/circlnc/). ${ }^{17}$ Gene ontology (GO) term and Kyoto Encyclopedia of Genes and Genomes (KEGG) pathway enrichment of co-expressed genes were analyzed by STRING (https://string-db.org/cgi/input.pl). The $P$ value $<0.05$ and gene count $\geq 3$ were set as the cut-off criteria.

\section{Plasmid Transient Transfection, Quantitative Real-Time PCR (qRT-PCR), Cell Counting Kit-8 (CCK-8) Assay, and Transwell Assay}

These methods were performed as described previously and are detailed in Supplementary Material (Table S7).

\section{Statistical Analysis}

All the analyses, including the heatmap, Cox regression analyses, $\mathrm{K}-\mathrm{M}$ survival curves, and ROC curves were performed by the $\mathrm{R}$ statistical software and SPSS 24.0 software. The $P$-value less than 0.05 was considered significant. Values are expressed as the mean \pm standard error of the mean (SEM) from three separate experiments, except for a special annotation. 


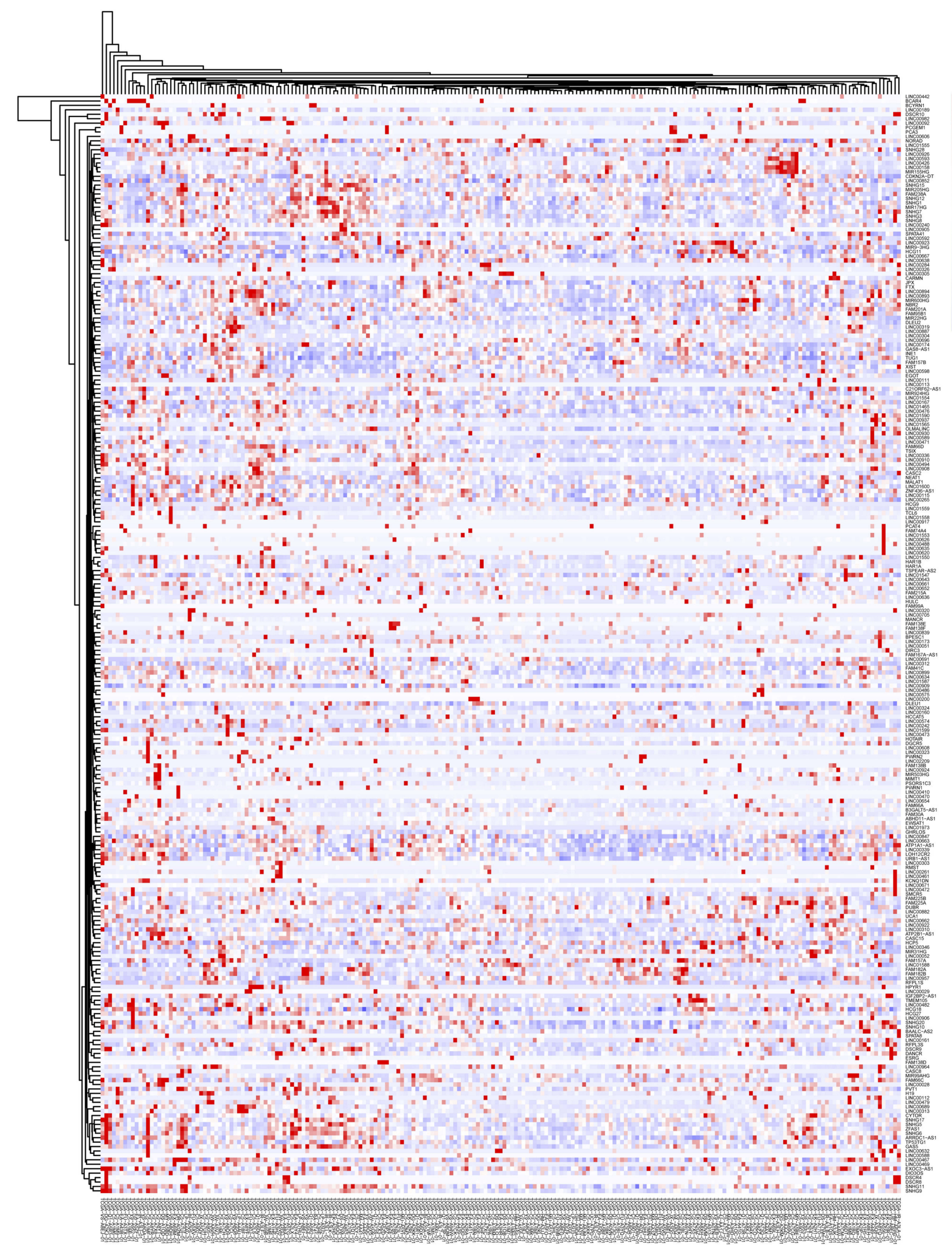

Figure I Differentially expressed IncRNAs in CC. The rows show 248 DELs, while the columns show 2 II samples. The red dot represents up-regulated IncRNAs, and blue dot represents down-regulated IncRNAs. 


\section{Results}

\section{Association of Demographic and Clinical Characteristics with RFS}

Based on the inclusion criteria, we screened 210 patients from the TCGA database at an age of $47.175 \pm 13.209$ years. Among them, there were $167 \mathrm{CC}$ subjects (79.1\%) with tumor stage I and II, and $39 \mathrm{CC}$ subjects (18.5\%) with tumor stage III and IV. Meanwhile, we examined the association of RFS and clinical features in CC patients by the univariate Cox regression analysis. The results suggested that lymph node metastasis (Hazard Ratio $(\mathrm{HR})=3.47, P=0.012$ ) was associated with RFS of CC patients (Table 1).

\section{Identification of DELs Associated with CC Recurrence}

A total of 4072 lncRNAs were extracted from the HGNC database (Table S1). The OncoPrint from the cBioPortal showed $248 \operatorname{lncRNAs}$ with expression alteration in more than $1 \% \mathrm{CC}$ patients based on Z-score with a threshold of \pm 2 (Table S2). Moreover, the expression alteration frequency of the 248 lncRNAs ranged from $1.4 \%$ to $16 \%$ in the CC patients with RNA-seq data (Figure 1).

Univariate Cox regression analysis revealed that 14 DELs, ie, LINC00905, LINC00173, LINC00189, HULC, CASC15, MIR22HG, HCG11, MIR924HG, LINC00852, FAM95B1, SNHG9, MALAT1, H19, and ARRDC1-AS1, among the 284 DELs had prominent prognostic values for
CC recurrence (Table 2). Afterward, the relationship between the 14 DELs and RFS was calculated by multivariate Cox regression analysis, and the results showed that four IncRNAs, ie, HCG11, CASC15, LINC00189, and LINC00905, were markedly associated with worse RFS of CC, whereas three lncRNAs, including HULC, LINC00173, and MIR22HG, were the opposite (Table 2 and Figure 2A-G). In addition, according to the cBioPortal, there were $61(29 \%)$ patients with altered expression of any one of the 7 DELs, including amplification, deep deletion, mRNA up-regulation and mRNA downregulation (Figure 3A). The genomic information of the 7 DELs is shown in Figure 3B. When all the 7 DELs were combined, their changes were also significantly associated with disease/progression-free survival and OS, respectively $(P=0.040$ and $P=0.007$; Figure $3 \mathrm{C}$ and $\mathrm{D})$.

\section{Prediction of CC Recurrence Risk by the RRS Based on DELs}

RRS was established to predict the recurrence risk of $\mathrm{CC}$ patients and defined as: $\mathrm{RRS}=\left(1.152 * \mathrm{EXP}_{\mathrm{HCG} 11}\right)$ $+\left(1.339^{*} \mathrm{EXP}_{\mathrm{CASC} 15}\right)+\left(1.646^{*} \mathrm{EXP}_{\mathrm{LINC00189}}\right)+$ $\left(3.939 * \mathrm{EXP}_{\mathrm{LINC} 00905}\right)+\left(-1.669 * \mathrm{EXP}_{\mathrm{HULC}}\right)+$ $\left(-1.982 * \mathrm{EXP}_{\mathrm{LINC00173}}\right)+\left(-1.446 * \mathrm{EXP}_{\mathrm{MIR} 22 \mathrm{HG}}\right)$. To evaluate the predictive power of the risk model, CC patients were classified into the low and high RRS groups based on the median RRS value (Figure 4A). Figure $4 \mathrm{~B}$ shows the RFS time and disease prognosis

Table 2 Prognostic Significance of DELs from Univariate and Multivariate Cox Regression Analysis

\begin{tabular}{|c|c|c|c|c|c|}
\hline \multirow[t]{2}{*}{ Gene Name } & \multicolumn{2}{|c|}{ Univariate Analysis } & \multicolumn{3}{|l|}{ Multivariate Analysis } \\
\hline & HR (95\% CI) & $P$ value & HR (95\% CI) & $\mathbf{P}$ value & $\boldsymbol{\beta}$ \\
\hline HCGII & $2.59(1.08-6.20)$ & 0.033 & $3.16(1.12-8.91)$ & 0.029 & 1.152 \\
\hline CASCI5 & $2.38(1.05-5.93)$ & 0.038 & $3.82(1.39-10.45)$ & 0.009 & 1.339 \\
\hline LINC00I89 & $2.32(1.02-5.25)$ & 0.044 & $5.19(1.84-14.60)$ & 0.002 & 1.646 \\
\hline LINC00905 & $4.77(1.4 I-16.17)$ & 0.012 & $51.36(8.79-300.29)$ & 0.000 & 3.939 \\
\hline HULC & $0.32(0.13-0.81)$ & 0.016 & $0.19(0.056-0.63)$ & 0.007 & -1.669 \\
\hline LINC00I73 & $0.32(0.13-0.77)$ & 0.011 & $0.15(0.05-0.46)$ & 0.001 & -1.928 \\
\hline MIR22HG & $0.32(0.13-0.80)$ & 0.015 & $0.23(0.07-0.75)$ & 0.015 & -1.466 \\
\hline MIR924HG & $2.47(1.03-5.92)$ & 0.043 & $2.37(0.82-6.85)$ & 0.110 & 0.864 \\
\hline LINC00852 & $0.37(0.16-0.87)$ & 0.022 & $0.45(0.15-1.36)$ & 0.156 & -0.795 \\
\hline FAM95BI & $0.32(0.14-0.78)$ & 0.011 & $0.50(0.17-1.47)$ & 0.205 & -0.700 \\
\hline SNHG9 & $0.33(0.14-0.79)$ & 0.013 & $0.51(0.17-1.52)$ & 0.228 & -0.671 \\
\hline MALATI & $0.30(0.12-0.76)$ & 0.011 & $0.68(0.24-1.92)$ & 0.468 & -0.383 \\
\hline $\mathrm{HI9}$ & $2.49(1.04-5.97)$ & 0.040 & $\mathrm{I} .46(0.48-4.47)$ & 0.509 & 0.377 \\
\hline ARRDCI-ASI & $0.42(0.18-0.97)$ & 0.043 & $0.89(0.35-2.26)$ & 0.809 & -0.115 \\
\hline
\end{tabular}

Note: Bold numbers indicate statistically significant differences with a $p$ value $<0.05$.

Abbreviations: HR, Hazard Ratio; $\mathrm{Cl}$, confidence interval; $\beta$, regression coefficient. 

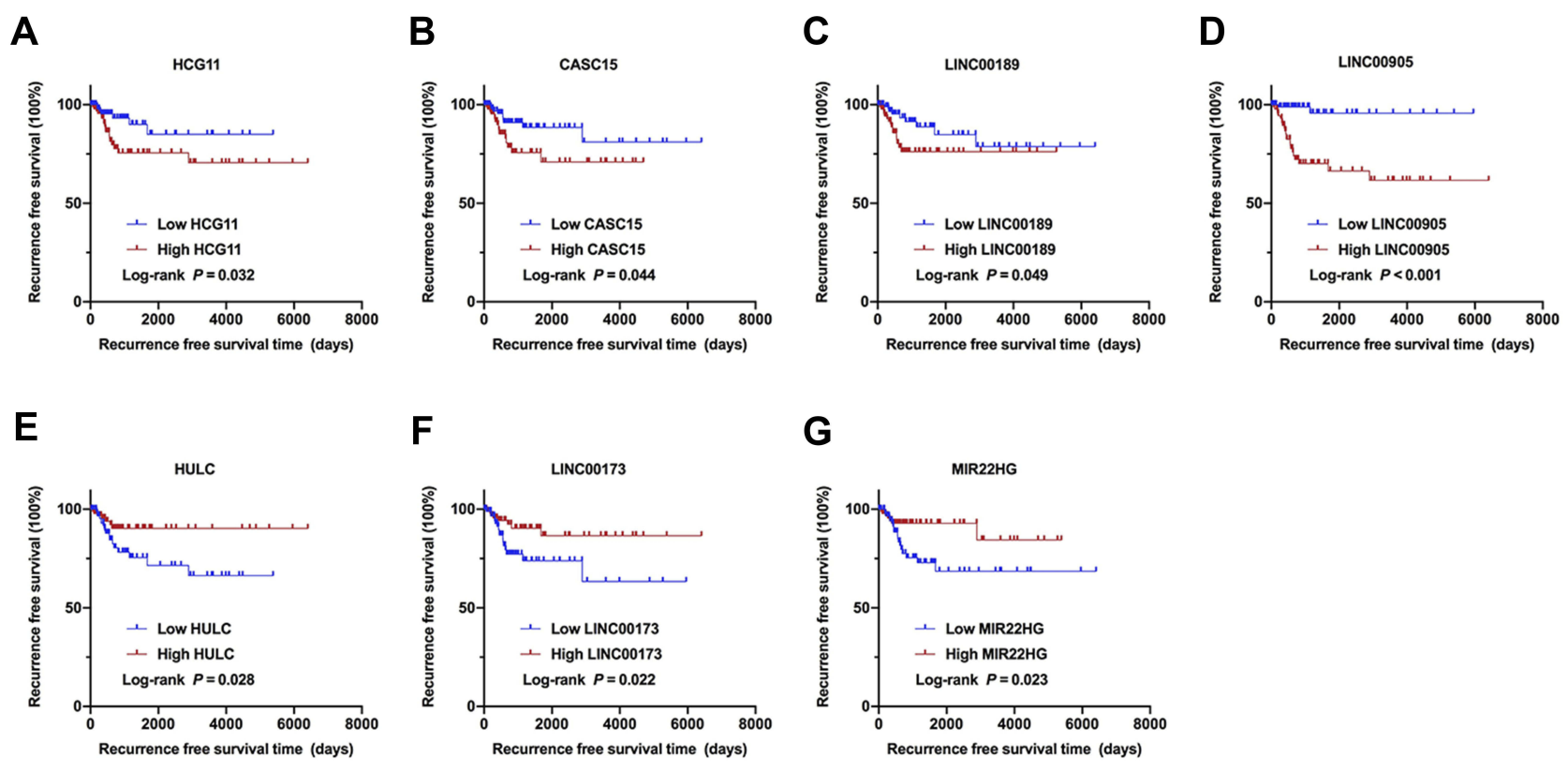

Figure 2 Identification of the 7 DELs associated with the recurrence of CC. (A-G) Kaplan-Meier survival curves of RFS in the TCGA cohort are shown according to HCGII, CASCI5, LINC00189, LINC00905, HULC, LINC00173, and MIR22HG expression, respectively.
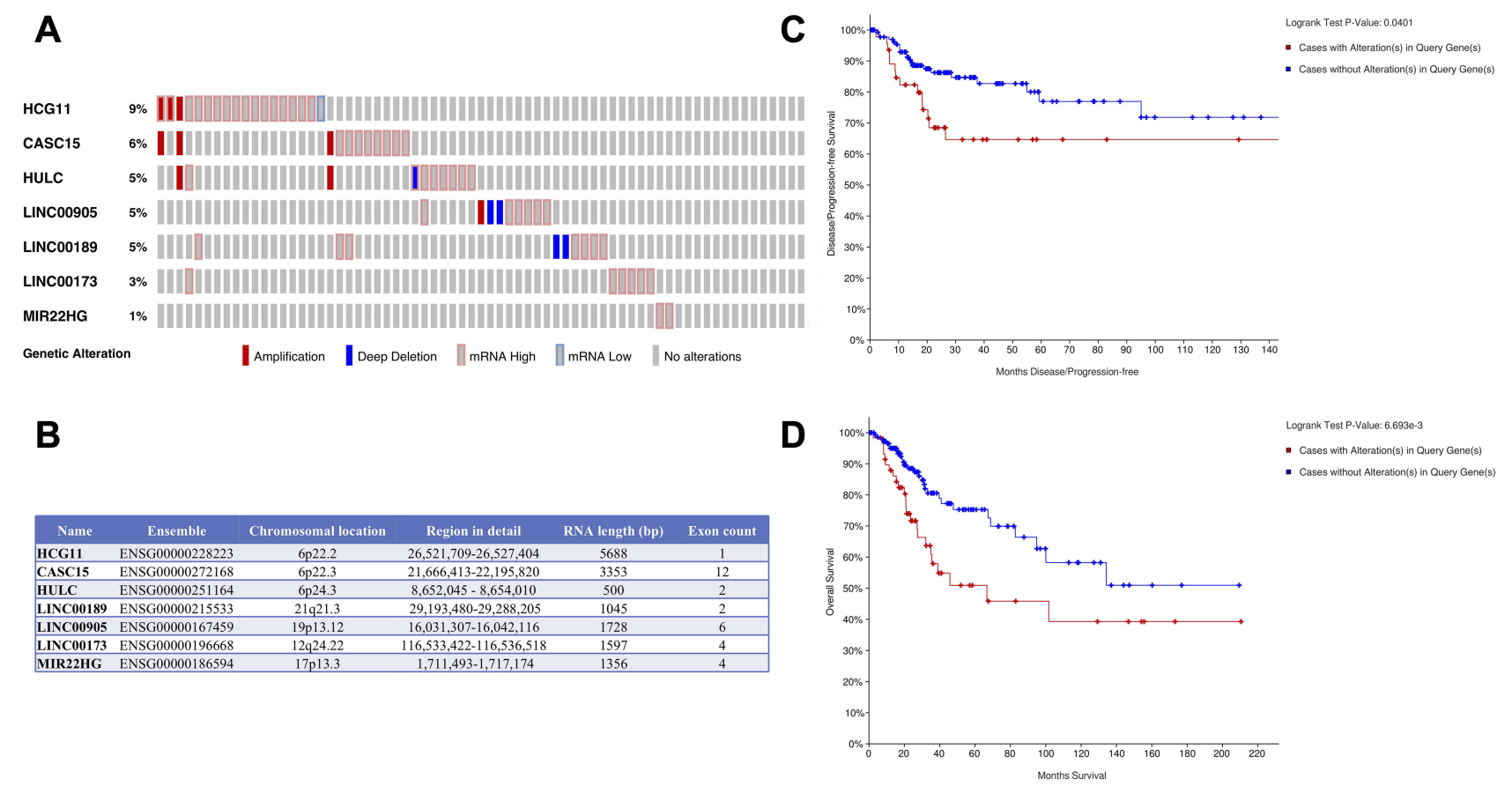

Figure 3 Identification of the 7 DELs signature associated with survival by cBioPortal. (A) The genetic expression alterations of the IncRNAs in CC. (B) Organizations of these 7 DELs and chromosome locations. (C) Kaplan-Meier curve for disease/progression-free survival based on alterations of the 7 DELs. (D) Kaplan-Meier curve for OS based on alterations of the 7 DELs.

for different RRS groups in CC patients. Furthermore, the expression pattern of the 7 DELs between the low and high RRS groups is shown in Figure 4C. The levels of HCG11, CASC15, LINC00189, and LINC00905 were remarkably higher in the high RRS group than that in the low RRS group, whereas three DELs, including HULC, LINC00173, and MIR22HG, were the opposite. K-M analysis revealed that the RFS time of the low RRS group was predominantly longer than that of the high RRS group $(P<0.001$; 


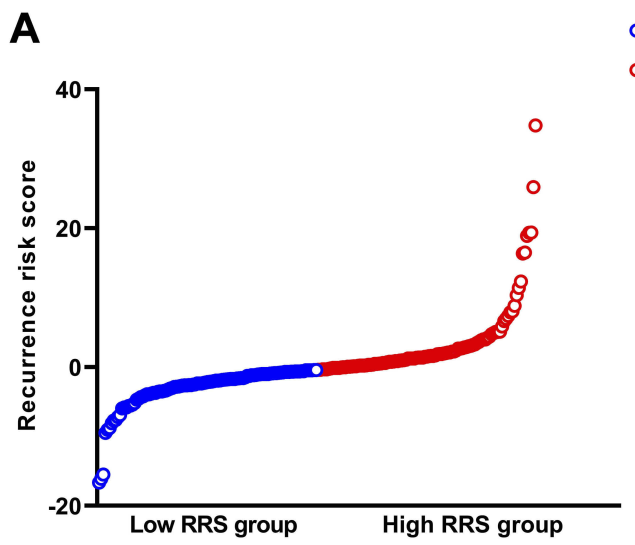

\section{B}

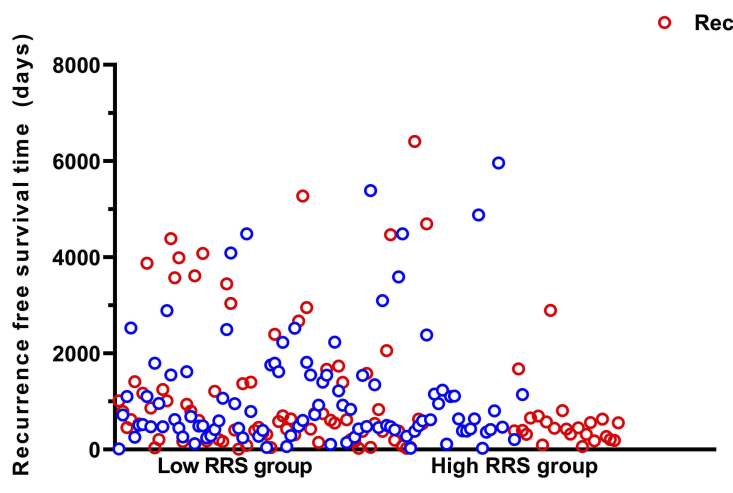

C

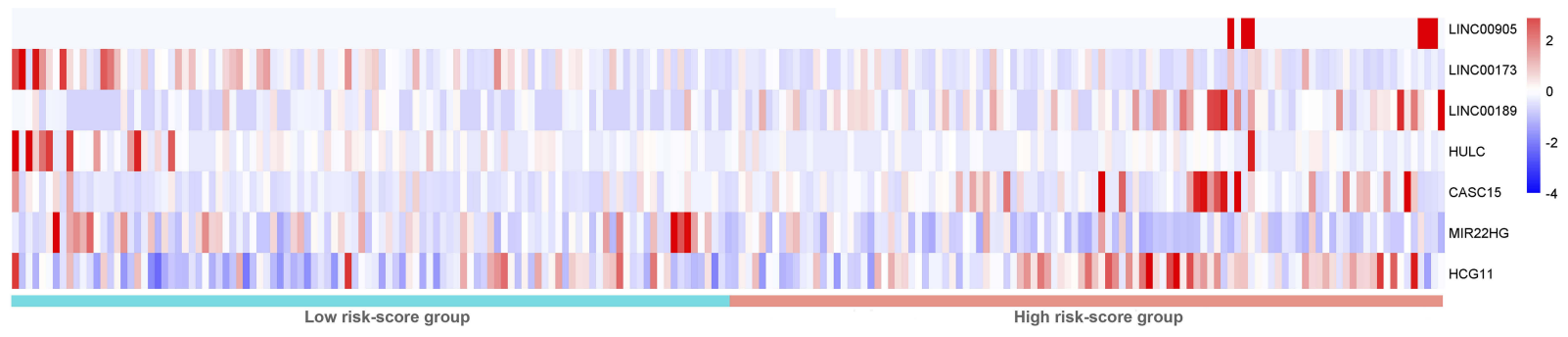

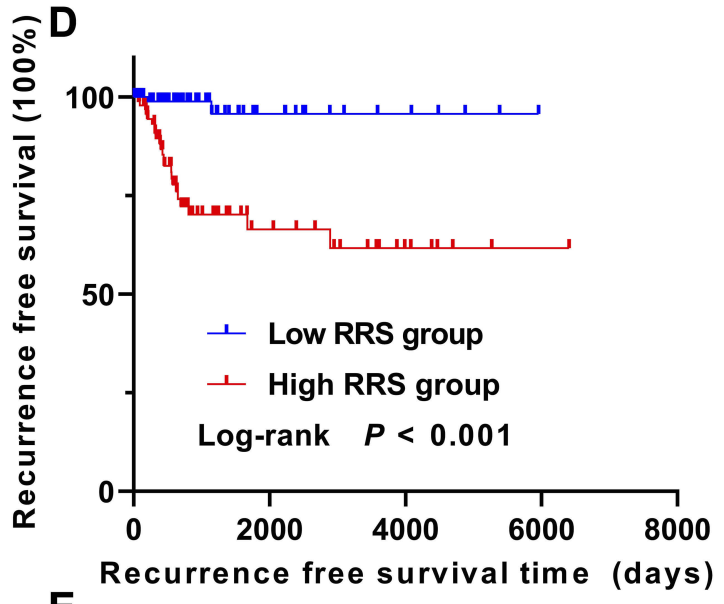

E

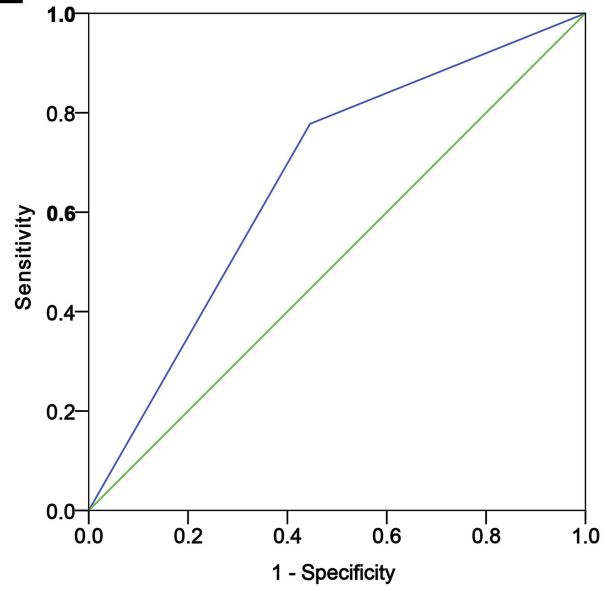

Figure 4 Prognostic value of the RRS constructed from 7 DELs in CC. (A) 7 DELs expression and RRS distribution by z-score. (B) Patients' recurrence status and RFS time; (C) Heatmap of the IncRNA expression profiles. Rows represent IncRNAs, and columns represent patients. The red dot represents up-regulated IncRNA, and blue dot represents down-regulated IncRNA. (D) Kaplan-Meier analysis of the RFS between high/low RRS groups. (E) Prognostic value of the RRS displayed as a time-dependent ROC curve for predicting the survival status. AUC, area under curve; $\mathrm{Cl}$, confidence interval.

Figure 4D). Meanwhile, the 7 DELs exhibited a well-predicted power of RFS for the CC patients, with an AUC value of $0.739(P<0.001$; Figure 4E).

\section{MIR22HG as a Key Recurrence-Related IncRNAs in CC}

We further investigated the difference of each lncRNA expression between the $\mathrm{CC}$ and matched normal tissues using the circlncRNAnet website, and found only the difference in HCG11 and MIR22HG expression between 

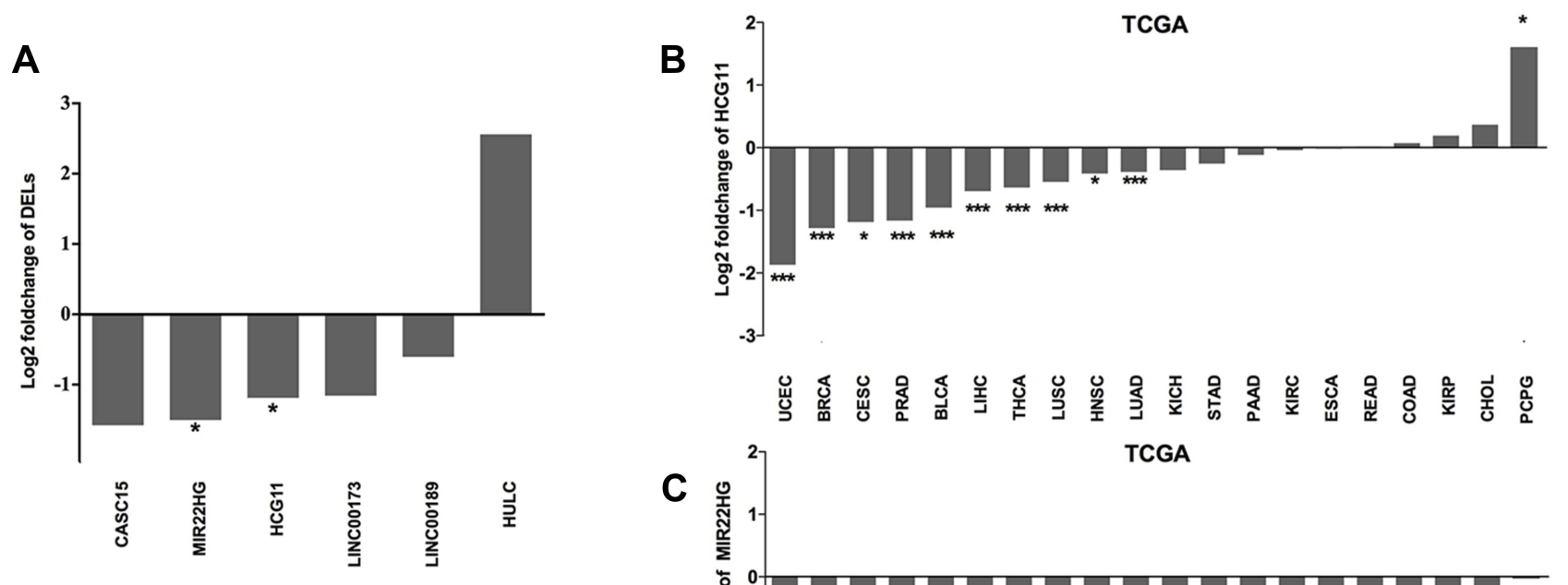

Figure 5 Relative expression of candidate IncRNAs from circlncRNAnet data. (A) The expression of DELs in tumor and adjacent tissues in CC. (B) Expression levels of HCGII in tumor and adjacent tissues in 20 cancers. (C) Expression levels of MIR22HG in tumor and adjacent tissues in 20 cancers. $* * *<0.00 \mathrm{I} ; * *<0,0 \mathrm{I} ; *<0.05$.

Abbreviations: BLCA, bladder urothelial carcinoma; BRCA, breast invasive carcinoma; CESC, cervical squamous cell carcinoma and endocervical adenocarcinoma; $\mathrm{CHOL}$, cholangiocarcinoma; COAD, colon adenocarcinoma; ESCA, esophageal carcinoma; HNSC, head and neck squamous cell carcinoma; KICH, kidney chromophobe; KIRC, kidney renal clear cell carcinoma; KIRP, kidney renal papillary cell carcinoma; LIHC, liver hepatocellular carcinoma; LUAD, lung adenocarcinoma; LUSC, lung squamous cell carcinoma; PAAD, pancreatic adenocarcinoma; PCPG, pheochromocytoma and paraganglioma; PRAD, prostate adenocarcinoma; READ, rectum adenocarcinoma; STAD, stomach adenocarcinoma; THCA, thyroid carcinoma; UCEC, uterine corpus endometrial carcinoma.

To examine the association of the HCG11 and MIR22HG signature with clinical parameters, $\mathrm{CC}$ patients were divided into low- and the high-expression group using the median of HCG11 or MIR22HG expression level as the cutoff value. Subgroup analysis demonstrated that increased MIR22HG was significantly relevant to decreased risks of recurrence among the subgroups of age at diagnosis $<45(\mathrm{HR}=0.26$, $P=0.044)$, stage $\mathrm{I} / \mathrm{II}(\mathrm{HR}=0.33, P=0.028), \mathrm{T}$ stage $\mathrm{I} / \mathrm{II}$ $(\mathrm{HR}=0.30, P=0.032)$, chemotherapy $(\mathrm{HR}=0.18$, $P=0.024)$, and molecular therapy $(\mathrm{HR}=0.16, P=0.018)$, suggesting it as a potential prognostic indicator of $\mathrm{CC}$ (Table 3). However, there was no marked correlation between HCG11 and clinical features (Table S5).

\section{Functional Enrichment Analysis of MIR22HG}

To acquire more information about the molecular functions and signal pathways of the lncRNAs that had been selected, the co-expressed genes of MIR22HG were predicted by circlncRNAnet. Online website STRING was used to analyze the GO term and KEGG pathway enrichment of the co-expressed genes. We selected the top 5 most significantly enriched GO terms (biological process (BP), cellular component, and molecular function) (according to the $P$-value) and the top 15 most significantly enriched KEGG pathways (Figure 6 and Table S6). The enrichment results indicated the involvement of MIR22HG in the regulation of cell cycle since the most significant BP term and KEGG pathway were "cell cycle G1/S phase transition" and "Cell cycle", respectively.

\section{Effects of MIR22HG on CC Cell Phenotypes}

To further investigate the biological function of MIR22HG in CC, we transfected the GV146/ MIR22HG vector into $\mathrm{HeLa}$ and $\mathrm{SiHa}$ cells, respectively (Figure 7A). Compared with the negative control (NC), HeLa and SiHa cells overexpressing MIR22HG had the lower proliferation $(P<0.05$; Figure $7 \mathrm{~B}$ and C). Transwell assays revealed that migration and invasion ability were suppressed in $\mathrm{CC}$ cells transfected with MIR22HG $(P<0.05$; Figure $7 \mathrm{D}$ and $\mathrm{E})$. In addition, to rule out the possibility that the inhibitory effect 
Table 3 Subgroup Survival Analyses for Correlation of Clinicopathological Characteristics and MIR22HG Expression in CC Patients

\begin{tabular}{|c|c|c|c|c|}
\hline \multirow[t]{2}{*}{ Variable } & \multicolumn{2}{|c|}{$\begin{array}{l}\text { MIR22HG } \\
\text { Expression } \\
\text { (Patients/ } \\
\text { Recurrence) }\end{array}$} & \multirow[t]{2}{*}{ HR $(95 \% \mathrm{Cl})$} & \multirow[t]{2}{*}{$P$ value } \\
\hline & Low & High & & \\
\hline \multicolumn{5}{|l|}{ Age at diagnosis } \\
\hline$<45$ & $41 / 8$ & $58 / 3$ & $0.26(0.07-0.96)$ & 0.044 \\
\hline$\geq 45$ & $57 / 10$ & $43 / 4$ & $0.53(0.17-1.69)$ & 0.282 \\
\hline \multicolumn{5}{|l|}{ Number of pregnancies } \\
\hline$\leq 3$ & $52 / 11$ & $53 / 3$ & $0.29(0.80-1.03)$ & 0.055 \\
\hline$>3$ & $36 / 5$ & $37 / 4$ & $0.76(0.20-2.82)$ & 0.675 \\
\hline \multicolumn{5}{|l|}{ Smoking status } \\
\hline No & $48 / 9$ & $50 / 4$ & $0.41(0.13-1.33)$ & 0.136 \\
\hline Yes & $42 / 7$ & $43 / 2$ & $0.26(0.05-1.26)$ & 0.095 \\
\hline \multicolumn{5}{|l|}{$\begin{array}{l}\text { Neoplasm histologic } \\
\text { grade }\end{array}$} \\
\hline $\mathrm{GI} / \mathrm{G} 2$ & $47 / 10$ & $56 / 3$ & $0.29(0.08-1.05)$ & 0.059 \\
\hline $\mathrm{G} 3 / \mathrm{G} 4$ & $41 / 8$ & $36 / 4$ & $0.53(0.16-1.77)$ & 0.304 \\
\hline \multicolumn{5}{|l|}{ Stage } \\
\hline I/II & $80 / 17$ & $77 / 5$ & $0.33(0.12-0.89)$ & 0.028 \\
\hline $\mathrm{III/IV}$ & $17 / 1$ & $20 / 2$ & $1.18(0.10-13.42)$ & 0.897 \\
\hline \multicolumn{5}{|l|}{ T stage } \\
\hline $\mathrm{Tl} / \mathrm{T} 2$ & $70 / 14$ & $70 / 4$ & $0.30(0.10-0.90)$ & 0.032 \\
\hline T3/T4 & $7 / 1$ & $4 / 1$ & $1.53(0.10-24.58)$ & 0.765 \\
\hline \multicolumn{5}{|l|}{ Lymph node metastasis } \\
\hline No & $44 / 5$ & $46 / 2$ & $0.42(0.08-2.16)$ & 0.297 \\
\hline $\mathrm{NI}$ & $19 / 7$ & $22 / 3$ & $0.34(0.09-1.34)$ & 0.124 \\
\hline \multicolumn{5}{|l|}{ Metastasis } \\
\hline Mo & $48 / 5$ & $34 / 2$ & $0.32(0.07-1.5 \mathrm{I})$ & 0.150 \\
\hline MI & $3 / 0$ & $5 / 0$ & & \\
\hline \multicolumn{5}{|l|}{ Chemotherapy } \\
\hline NO & $51 / 7$ & $51 / 5$ & $0.74(0.23-2.33)$ & 0.607 \\
\hline YES & $47 / 11$ & $50 / 2$ & $0.18(0.04-0.79)$ & 0.024 \\
\hline \multicolumn{5}{|l|}{ Radiation therapy } \\
\hline NO & $23 / 5$ & $32 / 2$ & $0.31(0.06-1.60)$ & 0.162 \\
\hline YES & $67 / 13$ & $63 / 5$ & $0.41(0.15-1.14)$ & 0.086 \\
\hline \multicolumn{5}{|l|}{ Molecular therapy } \\
\hline NO & $24 / 4$ & $25 / 5$ & $1.50(0.40-5.60)$ & 0.546 \\
\hline YES & $46 / 11$ & $52 / 2$ & $0.162(0.036-0.73)$ & 0.018 \\
\hline
\end{tabular}

Note: Bold numbers indicate statistically significant differences with a $\mathrm{p}$ value $<0.05$ Abbeviations: $\mathrm{HR}$, Hazard Ratio; $\mathrm{Cl}$, confidence interval.

of MIR22HG on CC cell phenotype is dependent on HPV infection, similar experiments with C-33 A cell, an HPV-cervical cancer cell line, were performed and led to similar conclusions (Figure S1A-D).

\section{Discussion}

Recurrent $\mathrm{CC}$ is almost always incurable and has no typical symptoms ${ }^{18}$ Considering the high mortality rate among recurrent CC patients, it is necessary to develop suitable prognosis biomarkers with the potential to predict tumor progression. The ability to predict which patients have a high risk of recurrence would empower clinicians to better patient therapy. ${ }^{19}$ LncRNAs have been reported as key regulators in the progression and metastasis of cancer. ${ }^{20}$ Compared with mutations or aberrant expression in protein-coding genes, the expression of lncRNAs was more tissue-specific. ${ }^{20,21}$ Given that many $\operatorname{lncRNAs}$ were critical for cancer progression and prognostic, we needed to deeply investigate that lncRNAs may possess an unknown function in cancer. ${ }^{22}$

In this study, we analyzed the lncRNAs expression profiles of patients with CC downloaded from TCGA and identified seven DELs (HCG11, CASC15, LINC00189, LINC00905, HULC, LINC00173, and MIR22HG) signature associated with RFS. Among the 7 DELs, the potential of some lncRNAs as prognostic-associated biomarkers for cancer has been demonstrated in many cancers. CASC 15 was up-regulated in $\mathrm{CC}$, and its overexpression associated with lymph node metastasis and FIGO stage, indicating a poor prognosis for $\mathrm{CC} .{ }^{23} \mathrm{HULC}$ was abnormally upregulated in a variety of cancers and meta-analysis indicated that overexpression of HULC was associated with metastasis and a poor OS in cancer. ${ }^{24}$ Zhang et $\mathrm{al}^{25}$ presented that low expression HCG11 in tissues was associated with poor survival of prostate cancer patients. Likewise, in our study, downregulation of HCG11 expression was associated with shorter RFS of CC patients. However, HCG11 was down-regulated in CC compared to adjacent normal tissues, which meant that our findings of HCG11 needed further study.

In addition to HCG11, the comparison of the 7 DELs expression between tumor and adjacent normal tissues in TCGA suggested that only MIR22HG could act as a suppressor gene in CC. Decreased MIR22HG expression also significantly increased the risk of recurrence among the subgroups of age at diagnosis $<45$, stage I/II, T stage I/II, chemotherapy, and molecular therapy while HCG11 failed to correlate with these clinicopathological features of CC accordantly. Emerging evidence suggested that MIR22HG was a tumor suppressor gene, which contributed to the initiation and progression of many cancers. Highly expressed MIR22HG inhibited cancer cell growth, migration and 
A

BP:

CC:

MF:

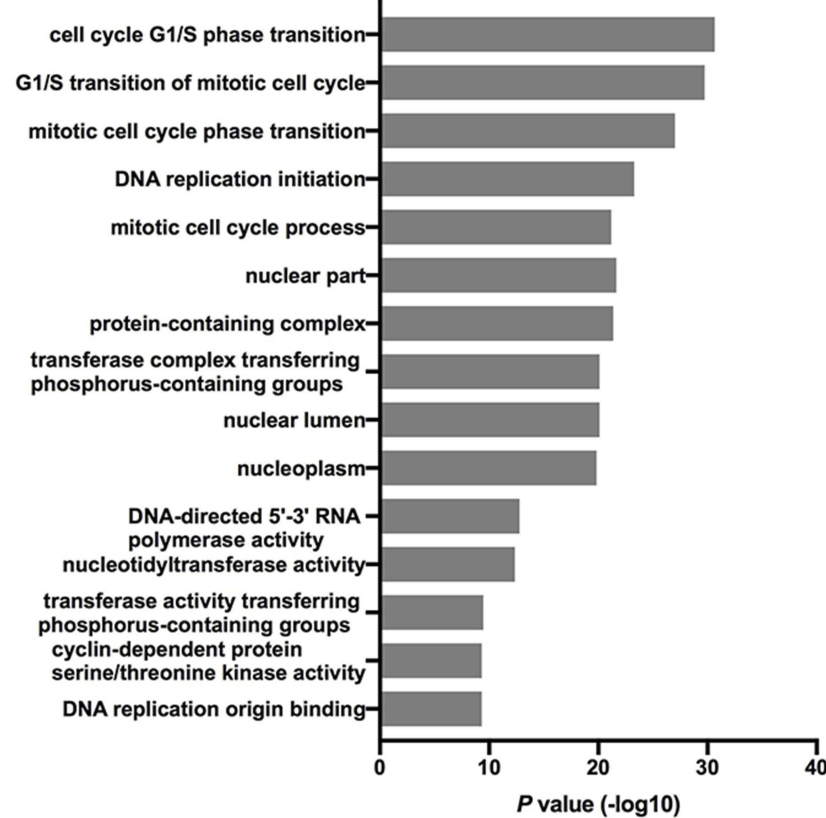

B

KEEG pathways

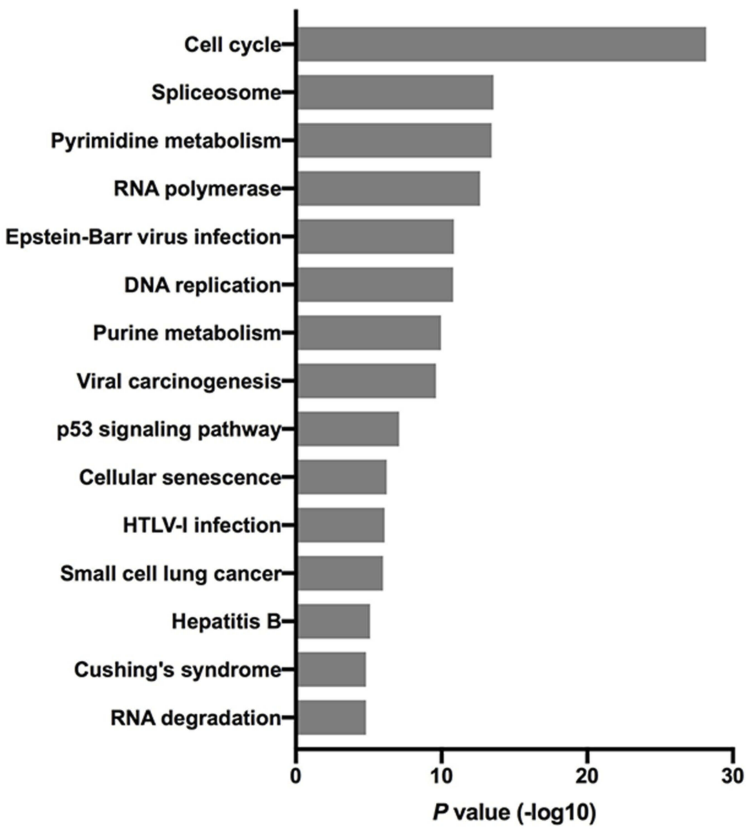

Figure 6 Enrichment of GO terms and KEGG pathways for co-expressed mRNAs of MIR22HG. (A and B) KEGG and GO analysis of the related genes. Abbreviations: GO, gene ontology; KEGG, kyoto encyclopedia of genes and genomes; BP, biological process; CC, cellular component; MF, molecular function.

A
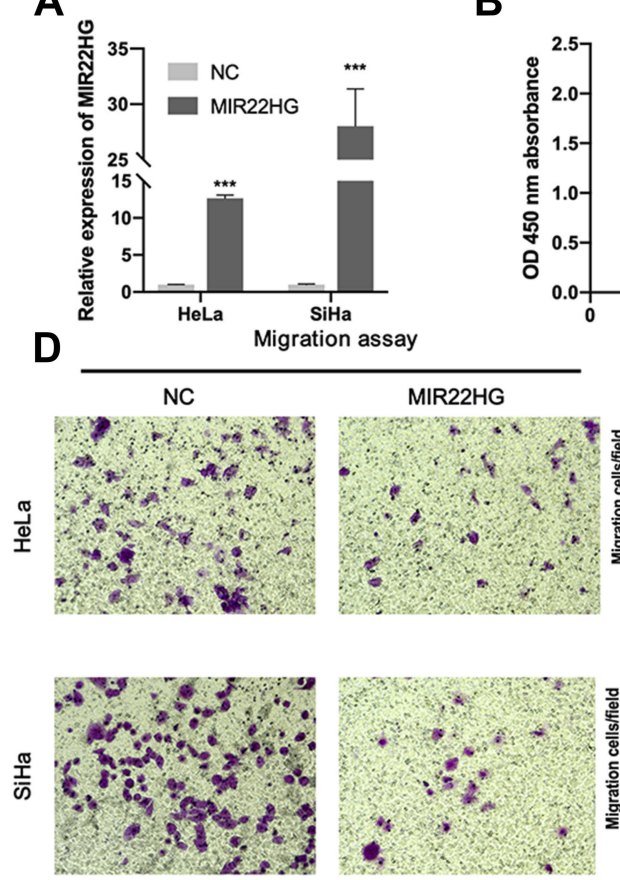

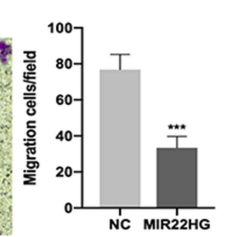

B

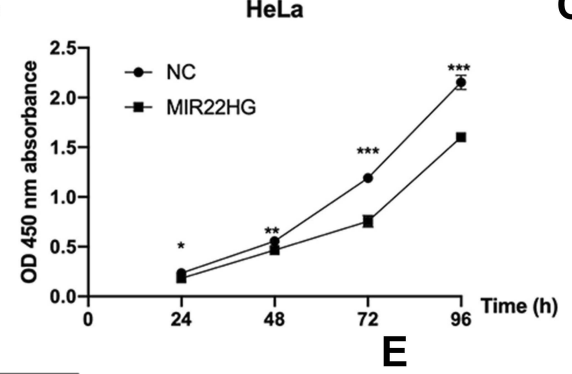

C
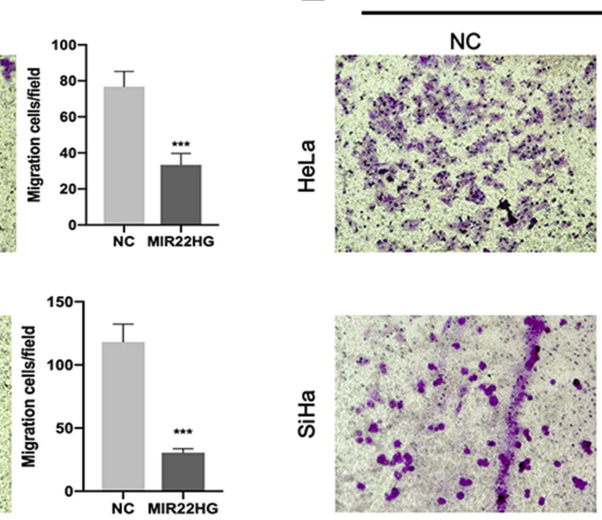

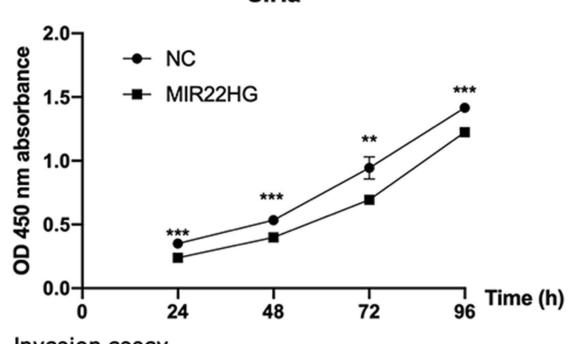

$\mathrm{SiHa}$
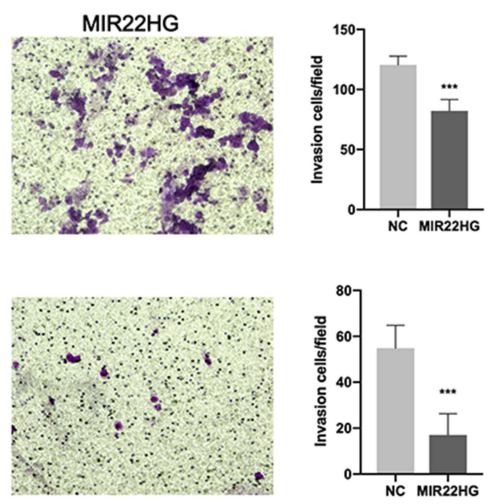

Figure 7 MIR22HG overexpression inhibited CC cells proliferation. (A) qRT-PCR determined expression efficiency of transfection with MIR22HG in HeLa and SiHa cells. Quantitative normalization of MIR22HG was performed in each sample using GAPDH expression as an internal control. (B and C) The CCK-8 assay was conducted to measure cell proliferation in HeLa and SiHa cells after transfection with MIR22HG. (D and E) Transwell assays were used for testing the migration and invasion ability of HeLa and SiHa cells after transfection with MIR22HG (I0X). ***P<0.00I, **P<0.0I, $* \mathrm{P}<0.05$. 
invasion, and overexpression MIR22HG had a better prognosis in HCC patients. $^{26}$ The pathways that MIR22HG may mediate in $\mathrm{CC}$ remain unclear, so we performed functional enrichment analysis to identify MIR22HG-associated biological signaling pathways. The GO BP term and KEGG pathway most significantly associated with MIR22HG were "cell cycle G1/S phase transition" and "Cell cycle", respectively. Functionally, elevated MIR22HG expression could suppress $\mathrm{CC}$ cell proliferation, migration and invasion. Those data indicated that MIR22HG could play important roles in CC recurrence. Cell-cycle dysregulation was an important indicator of tumor development. Cui et $\mathrm{al}^{27}$ reported that MIR22HG was downregulated in endometrial cancer, which inhibited endometrial cancer cell proliferation and arrested cells in $\mathrm{G} 0 / \mathrm{G} 1$ phase to promote apoptosis by regulating miR-141-3p/DAPK1 axis. The role of MIR22HG in the recurrence of $\mathrm{CC}$ warrants further investigation. Except for those IncRNAs mentioned above, LINC00189, LINC00905 and LINC00173 are currently less studied, which imply that the biological functions of these lncRNAs remain to be explored.

Thus far, the studies on the role of IncRNAs in the recurrence of $\mathrm{CC}$ were limited and our findings may have some clinical implications. However, there were some limitations in this study. First, only a few lncRNAs (4072 lncRNAs extracted from the HGNC database) were investigated this time, so many lncRNAs which are not received in the HGNC database are not determined in the present study; second, the demographic data of patients in the TCGA may not represent those of other patient populations; third, we were not capable to validate the findings using our patients' cohort due to insufficient follow-up information; finally, the molecular mechanisms of the 7 DELs were unclear and further functionality studies were needed to better understand the roles of 7 DELs in the recurrence of CC.

In summary, this study reported a seven-lncRNA signature to predict recurrence in $\mathrm{CC}$ patients by comprehensive analysis of lncRNAs expression profiles in the TCGA database. Especially, MIR22HG might be a potential prognostic indicator for $\mathrm{CC}$. We have shown that MIR22HG could suppress $\mathrm{CC}$ cells proliferation, metastasis and invasion. However, future functional investigations are required to further explore the mechanisms underlying the roles of these IncRNAs in $\mathrm{CC}$ recurrence.

\section{Acknowledgments}

This study was supported by Natural Science Foundation of China (81872684), Natural Science Foundation of Jiangsu
Province (BK20171367), the Six Talent Peaks Project in Jiangsu Province (WSW-201), the "SIX ONE" Talent Research Project for the High-level Health Personnel of Jiangsu Province (LGY2018037), Nantong Municipal Science and Technology Plan (JC2018053 and YYZ17072), Nantong Municipal Key Talent Project (No. 9), and funded by Southeast University "Zhongying Young Scholars” Project.

\section{Disclosure}

All authors declare that they have no conflicts of interest in this work.

\section{References}

1. Small WJ, Bacon MA, Bajaj A, et al. Cervical cancer: a global health crisis. Cancer. 2017;123(13):2404-2412. doi:10.1002/cncr.v123.13

2. Zhu FC, Hu SY, Hong Y, et al. Efficacy, immunogenicity and safety of the AS04-HPV-16/18 vaccine in Chinese women aged 18-25 years: end-of-study results from a Phase II/III, randomised, controlled trial. Cancer Med. 2019:8(14):6195-6211.

3. Sankaranarayanan R, Swaminathan R, Brenner H, et al. Cancer survival in Africa, Asia, and Central America: a population-based study. Lancet Oncol. 2010;11(2):165-173. doi:10.1016/S14702045(09)70335-3

4. Friedlander M. Guidelines for the treatment of recurrent and metastatic cervical cancer. Oncologist. 2002;7(4):342-347.

5. Quinn MA, Benedet JL, Odicino F, et al. Carcinoma of the cervix uteri. FIGO 26th annual report on the results of treatment in gynecological cancer. Int J Gynaecol Obstet. 2006;95(Suppl 1):S43-S103. doi:10.1016/S0020-7292(06)60030-1

6. Petignat P, Roy M. Diagnosis and management of cervical cancer. BMJ. 2007;335(7623):765-768. doi:10.1136/bmj.39337.615197.80

7. Quinn JJCH, Chang HY. Unique features of long non-coding RNA biogenesis and function. Nat Rev Genet. 2016;47-62. doi:10.1038/ nrg. 2015.10

8. Gao YF, Wang ZB, Zhu T, et al. A critical overview of long non-coding RNA in glioma etiology 2016: an update. Tumour Biol. 2016;37(11):14403-14413. doi:10.1007/s13277-016-5307-4

9. Cheetham SW, Gruhl F, Mattick JS, Dinger ME. Long noncoding RNAs and the genetics of cancer. Br J Cancer. 2013;108 (12):2419-2425. doi:10.1038/bjc.2013.233

10. Hoppe-Seyler F, Hoppe-Seyler K. Emerging topics in human tumor virology. Int J Cancer. 2011;129(6):1289-1299. doi:10.1002/ijc.26087

11. Peng L, Yuan X, Jiang B, Tang Z, Li GC. LncRNAs: key players and novel insights into cervical cancer. Tumour Biol. 2016;37 (3):2779-2788. doi:10.1007/s13277-015-4663-9

12. Li J, Wang Y, Yu J, Dong R, Qiu H. A high level of circulating HOTAIR is associated with progression and poor prognosis of cervical cancer. Tumour Biol. 2015;36(3):1661-1665. doi:10.1007/ s13277-014-2765-4

13. Zheng Z, Gao Y. Down-regulation of lncRNA snaR is correlated with postoperative distant recurrence of HPV-negative cervical squamous cell carcinoma. Biosci Rep. 2018;38(6). doi:10.1042/BSR20181213

14. Zhang J, Lin Z, Gao Y, Yao T. Downregulation of long noncoding RNA MEG3 is associated with poor prognosis and promoter hypermethylation in cervical cancer. J Exp Clin Cancer Res. 2017;36(1):5. doi:10.1186/s13046-016-0472-2

15. Cerami E, Gao J, Dogrusoz U, et al. The cBio cancer genomics portal: an open platform for exploring multidimensional cancer genomics data. Cancer Discov. 2012;2(5):401-404. doi:10.1158/21598290.CD-12-0095 
16. Wang $\mathrm{K}$, Li J, Xiong YF, Zeng Z, Zhang X, Li HY. A potential prognostic long noncoding RNA signature to predict recurrence among ER-positive breast cancer patients treated with tamoxifen. Sci Rep. 2018;8(1):3179. doi:10.1038/s41598-018-21581-w

17. Wu SM, Liu H, Huang PJ, et al. circlncRNAnet: an integrated web-based resource for mapping functional networks of long or circular forms of noncoding RNAs. GigaScience. 2018;7(1):1-10.

18. Tempfer CB, Beckmann MW. State-of-the-art treatment and novel agents in local and distant recurrences of cervical cancer. Oncol Res Clin Cancer Res. 2016;39(9):525-533. doi:10.1159/000448529

19. Bodurka-Bevers D, Morris M, Eifel PJ, et al. Posttherapy surveillance of women with cervical cancer: an outcomes analysis. Gynecol Oncol. 2000;78(2):187-193. doi:10.1006/gyno.2000.5860

20. Bhan A, Soleimani M, Mandal SS. Long noncoding RNA and cancer: a new paradigm. Cancer Res. 2017;77(15):3965-3981. doi:10.1158/ 0008-5472.CAN-16-2634

21. Fatima R, Akhade VS, Pal D, Rao SM. Long noncoding RNAs in development and cancer: potential biomarkers and therapeutic targets. Mol Cell Ther. 2015;3:5.

22. Gao P, Wei GH. Genomic insight into the role of lncRNA in cancer susceptibility. Int J Mol Sci. 2017;18(6):1239.
23. Shan S, Li HF, Yang XY, et al. Higher lncRNA CASC15 expression predicts poor prognosis and associates with tumor growth in cervical cancer. Eur Rev Med Pharmacol Sci. 2019;23(2):507-512. doi:10.26355/eurrev_201901_16862

24. Ding Y, Sun C, Li J, et al. The significance of long non-coding RNA HULC in predicting prognosis and metastasis of cancers: a meta-analysis. Pathol Oncol Res. 2019;25(1):311-318. doi:10.1007/ s12253-017-0351-y

25. Zhang Y, Zhang P, Wan X, et al. Downregulation of long non-coding RNA HCG11 predicts a poor prognosis in prostate cancer. Biomed Pharmacother. 2016;83:936-941. doi:10.1016/j. biopha.2016.08.013

26. Wu Y, Zhou Y, Huan L, et al. LncRNA MIR22HG inhibits growth, migration and invasion through regulating the miR-10a-5p/NCOR2 axis in hepatocellular carcinoma cells. Cancer Sci. 2019;110 (3):973-984. doi:10.1111/cas.13950

27. Cui Z, An X, Li J, Liu Q, Liu W. LncRNA MIR22HG negatively regulates miR-141-3p to enhance DAPK1 expression and inhibits endometrial carcinoma cells proliferation. Biomed Pharmacother. 2018;104:223-228. doi:10.1016/j.biopha.2018.05.046

\section{Publish your work in this journal}

Cancer Management and Research is an international, peer-reviewed open access journal focusing on cancer research and the optimal use of preventative and integrated treatment interventions to achieve improved outcomes, enhanced survival and quality of life for the cancer patient.
The manuscript management system is completely online and includes a very quick and fair peer-review system, which is all easy to use. Visit http://www.dovepress.com/testimonials.php to read real quotes from published authors. 